\title{
CONSIDERACIONES SOBRE LA MEDIDA DEL TIEMPO*
}

\section{HONORATO DE CASTRO. (**)}

Cuando nos encontramos ante uno de esos llamadas cuadrantes solares y vemos que la sombra proyectada por el estilete coincide con una de las láminas dibujadas, por ejemplo, con aquella en que aparece la inscripción de las $\mathrm{XI}$, acostumbramos a decir: - "Son las once de la mañana".- Y hasta es posible que encontremos una coincidencia entre las horas señaladas por el cuadrante solar y por nuestro reloj de bolsillo. Pero si tal coincidencia se produjo, tened la seguridad de que, si volvemos al siguiente día y tenemos la fortuna de poseer un reloj de bolsillo que ni se adelante ni se retrase un solo segundo, nos encontraremos ante un fenómeno que desconocen tan sólo quienes carecen de los conocimientos más rudimentarios acerca de los movimientos aparentes del sol. Nuestro reloj estark adelantado o retrasado con relación $\alpha$ las indicaciones del cuadrante solar. $\mathrm{Y}$ si hemos encontrado coincidencia en las indicaciones de uno y otro instrumento en un cierto día del año, en el transcurso del mismo habrá otro día más y probablemente tres en los que las indicaciones de nuestro reloj coincidirán con las del cuadrante solar.

La razón es bien conocida: es que la hora de nuestro reloj es hora oficial mientras que la señalada por el cuadrante solar es hora solar verdadera. No vayamos por ello a creer, por contraposición al adjetivo verdadero, que la hora oficial es una hora falsa. Nada de eso. Tan verdaderas son las indicaciones de un instrumento como las del otro. Lo que sucede es que, $\alpha$ conceptos diferentes, corresponden nombres diferentes; si la denominación ha de ser correcta, los nombres deben guardar una relacion estrecha con las cosas nominadas. Y como para contar el tiempo nos sirve unas veces como elemento de referencia el Sol verdadero $\mathrm{y}$, otras veces un Sol ficticio o ima-

(*) Original recibido en enero de 1958

(**) Geofísico; Gerencia de Exploración de Petroleos Mexicanos 
ginario, sin existencia real, de aquí resulta adecuado el llamar tiempo $u$ hora verdadera a la que se regula por el Sol verdadero, para distinguirla de la llamada hora media que se regula por el Sol medio o Sol ficticia a que hemos hecho referencia.

Antes de que hablemos de la hora oficial de México conviene que precisemos un poco los conceptos de hora Eolar verdadera, de hora solar media y de que veamos cual es el significado de las índicaciones del cuadrante solar.

Tanto la hora solar verdadera como la hora solar media son horas locales por estar una y otra referidas al meridiano de la localidad.

En un lugar cualquiera comienza el día solar verdadero astronb́mico cuando el Sol verdadero pasa por el meridiono de dicho lugar. El día civil comienza doce horas antes que el astronómico, o sea, cuando el Sol verdadero pasa por el antimeridiano. También se acostumbran a expresar de otra manera los momentos de comienzo de los días astronómico y civil diciendo que comienza el astronómico on el momento del paso superior del Sol por el meridiano de la localidad, y comienza el día civil en el momento del paso inferior por el mismo meridiano.

El fin del día astronómico o del día civil tendrá lugar cuando el Sol vuelva $a$ pasar por el meridiano o antimeridiano que sirvió para fijar su comienzo.

La duración del día solar verdadero no es constante; varía de un día a otro; unas veces crece y otras decrece. Por ello no podemos utilizar el día solar verdadero para medir el tiempo, porque es condición inherente a toda unidad de medida el ser de magnitud invariable.

Para fines astronómicos se utiliza una unidad de tiempo de valor constante, el día sidéreo que no sirve para fines de la vida corriente. Es el día sidéreo, el intervalo empleado por la Tierra para dar una vuelta completa en torno de su eje, es decir, para girar un ángulo de 360 grados. Pero es un poco más corto que el día solar y por ello no puede servirnos para los usos civiles. Entre la duración de uno y otro hay una diferencia de tres minutos con cincuenta y seis segundos (cua- 
tro minutos aproximadamente). Si en una fecha cualquiera comienzan al mismo tiempo el día sidéreo y el solar, el primero terminará cuatro minutos antes que el segundo; al cabo de un mes el día sidéreo llevará un adelanto de dos horas sobre el día solar.

Si pues acortásemos la longitud del péndulo que acciona nuestros relojes para que caminando más de prisa marcasen tiempo sidéreo, al cabo de un año habríamos vivido un día más, pero nuestras ocupaciones habituales deberian ser ejecutadas en horas con desigual iluminación puesto que sería de día en una época y de noche seis meses después.

No podemos pues utilizar el día sidéreo para los usos corrientes de la vida a pesar de la ventaja de ser una unidad constante. Pero tampoco podemos servirnos del día verdadero por no ser éste de duración invariable. Y sin embargo tenemos que ajustar nuestras actividades, a los movimientos acarentes del Sol, al igual que sucede con todos los seres vivien.. tes que pueblam nuestro planeta.

El conflicto se ha resuelto apelando a un Sol inexistente, a un Sol ficticio llamado Sol medio que, sin apartarse mucho del Sol verdadero, se mueve aparentemente sobre el ecuador mientras el verdadero va recorriendo aparentemente el plano de la órbita de la Tierra, el plano de la Eclíptica.

El Sol medio dá ocasión a considerar la unidad de tiem. po día medio de valor constante que comienza en cada lugar cuando pasa por el meridiano, o por el antimeridiano, el Sol medio, según que se considere el día astronómico a el día civil y que terminan cuando se produce ell paso subsiguiente.

La unidad de tiempo día medio tiene, como toda unidad de medida, sus divisores. Son éstos la hora, el minuto y el segundo de tiempo medio que diliferen en extensión de la hora, minuto y segundo de tíempo sidéreo. Se comprende fácilmente esta desigualdad si se tiene en cuenta que las unidades principales día sidéreo y día medio tienen distinta duración. En un día sidéreo la Tierra ha girado un ángulo de 360 grados y en un día medio el ángulo que la Tierra giró es un po- 
co mayor. El día sidéreo representa el intervalo de tiempo que transcurre entre dos pasos consecutivos por el meridiano de un lugar de un punto fijo de la esfera celeste, mientras que el día medio representa el intervalo de tiempo que ha transcurrido entre dos pasos consecutivos del Sol medio por el meridiano y es de tener en cuenta que este Sol medio no es un punto fijo de la esfera celeste sino que es un punto hipotético que se mueve a través de la misma y paralelamente al ecuador con un movimiento uniforme. En consecuencia un intervalo de tiempo no se exprescró por el mismo número cuando para representarlo se utilice el tiempo sidéreo que cuando se utilice el tiempo medio.

El comienzo del día sidéreo tiene lugar en cada localidad cuando pasa por su meridiano un punto especial de la esfera celeste que se eligió arbitrariamente: el llamado punto Aries, - punto equinoccial de la primavera, que es uno de los dos puntos de intersección de la eclíptica con el ecuador. En 24 horas de tiempo sidéreo el punto Aries se habrá separado del meridiano en un ángulo de 360 grados y como el movimiento de rotación de la Tierra es uniforme, en una hora de tiempo sidóreo, el punto Aries se habrór separado del meridiano un ángulo de 15 grados. Hablaríamos cnn más propiedad si dijéramos que es el meridiano, arrastrado por el movimiento de rotación de la Tierra, quien se separa del punto Aries. Así pues, cuando después de haber pasado por el meridiano el punto Aries, haya girado la Tierra un ángulo de 15 grados, habrá transcurrido una hora de tiempo sidéreo y diremos que en aquella localidad es la una, o que es una hora de tiempo sidéreo. Cuando el ángulo de gira de la tierra harya sido de 30 grados diremos que son las dos. Si consideramos el plano determinado por el punto Áries y el eje de rotación de la Tierra, y fijamos nuestra atención en el ángulo que el refe. rido plano forma con el meridiano de la localidad, ángulo que recibe el nombre de ángulo horcrio del punta Fries, veremos que es una magnitud que crece de una manera uniforme y contínua y que cuando un reloj regulado en tiempo sidéreo marque una hora cualquiera, por ejemplo las dos, el án- 
gulo horario antes mencionado valdrá treinta grados. Por ello es costumbre definir la hora sidérea de una localidad diciendo que es el ángulo horario occidental del Punto Aries.

Algo análogo sucede cuando en lugar de considerar como elementa de relerencia para la medida del tiempo al punto Aries que nos da la hora en tiempo sidéreo, consideramos al Sol medio por medio del cual regulamos nuestra vida con arreglo al tiempo medio. Así pues diremos que hora media de un lugar es el ángulo horario occidental del Sol medio. Y diremos que es la una, que son las dos de tiempo medio en un lugar, cuando el ángulo horario bccidental del punto ocupado por esa entidad imaginaria que llamamos Sol medio vale 15 grados, vale 30 grados, en ese mismo lugar.

Es preciso fijarse bien en que, en el intervalo de una hora de tiempo medio, la Tierra habrá girado un ángulo distinto de 15 grados, aunque sea esa la variación del valor del ángulo horario del Sol medio en ese intervalo de tiempo, porque estando el ángulo horario formado por dos planos (el plano del meridiano del lugar $y$ i el plano de círculo horario del astro), la variación de su valor puede obedecer a que cambien de posición uno o los dos planos que forman el ángulo horario. En el caso de la hora sidérea, como el plano determinado por el punto Aries y el eje del mundo es fijo, la variación del ángulo horario se produce tan sólo por el movimiento del meridiano que es arrastrado por la rotación de la Tierra; gira ésta por consecuencia 15 grados en una hora de tiempo sidéreo. Pero en el caso de la hora media se mueve al meridiano por la rotación de la Tierra y se mueve además el Sol medio. Por ello para que el Sol medio se separe del meridiano 15 grados, es decir, para que su ángulo horario alcance el valor de 15 grados, será preciso que transcurra un tiempo mayor, o sea, un poco más de una hora de tiempo sidéreo. Por ello es mayor la hora de tiempo medio que la de tiempo sidéreo.

De manera semejante llamamos hora solar verdadera de una localidad al ángulo horarlo occidental del Sol verdadero y diremos que en ese lugar son las dos o las tres horas de 
tiempo solar verdadero astronómico cuando el ángulo horario occidental del Sol verdadero tenga por valor en ese luga: treinta grados o cuarenta y cinco grados respectivamente.

Si el movimiento aparente del Sol verdadero a través de la esfera celeste fuese uniforme y paralelo al ecuador, entonces las horas solares verdaderas serían siempre iguales unas $\alpha$ otras. Pero como no se cumplen las condiciones antedichas, resulta que si medimos hoy con una unidad de tiempo constante el intervalo que transcurre entre las diez y las once, por ejemplo, y repetimos la operación en días sucesivos encontraremos valores diferentes. Por ello hemos necesitado acudir para medir el tiempo a considerar el Sol medio.

Una dificultad grave nos sale al paso en esta cuestión del tiempo medio. Es muy fácil decir que en un lugar comienza el día medio cuando el Sol media pasa por el meridiano. Pero cómo sabemos que se verifica ese fenómeno del comienzo siendo como es el Sol me dio un punto imaginario sin existencia real y al que por I onsecuencia no podemos bbservar. Cierto es en efecto que no podemos observar el Sol medio, pero podemos observar las estrellas que nos permiten conocer el tiempo verdadero, y conocidos estos elementos podemos mediante las reglas deducidas por estudios astronómicos pasar del conocimiento del tiempo sidéreo o del tiempo solar astronómico verdadero al del tiempo solor medio.

Dejando para otra ocasión el problema de pasar de tiempo sidéreo a tiempo medio, vamos a tratar de resolver la otra cuestión, la de pasar de tiempo solar verdadero a tiempo me. dio.

La transformación se realiza a través de la llamada ecuación de tiempo que no es ctra cosa que la cantidad de tiempo que hay que sumar a la hora verdadera para obtener la hora media. Todos los anuarios astronómicos publican su valor para el comienzo de todos los días del año $\mathrm{y}$, a veces, para todas los horas de cada uno de los días. Con una tabla a la vista la operación es bien sencilla. Pero como no siempre se tienen a mano las precitadas tablas se acostumbra en muchas 
ocasiones a construir una gráfica que permita par interpolación conocer su valor en cualquier momento del año.

Uno de los sitios más apropiados para la construcción de esta gráfica es sin duda el emplazamiento de un cuadrante solar, pues uno y otra se complementan para resolver el problema de conocer la hora media local.

Algo habremos ganado si llegamos a conocer la hora media local, pero nuestro problema quedará sin resolver porque no podemos vivir con hora local. Tenemos que eliminar la confusión enorme que resultaría del hecho de que cada ciudadano tuviese ajustado su reloj al meridiano de su vecindad, porque en tal caso tendría que cambiar de hora al cambiar de residencia y el desarreglo en los servicios públicos sería enorme. Hemos de vivir con arreglo a la llamada hora oficial que es distinta para cada uno de los veinticuatro usos horarios en que la superficie terrestre ha sido dividida. Los usos horarios estón limitados por meridianos terrestres que forman óngulos de 15 grados. Todos los paises que están comprendidos dentro de un huso tienen la misma hora oficial; en todos ellos comienza el día cuando el Sol medio pasa por el antimeridiono central del huso. Las horas de paises situados en husos contiguos difieren en una hora exacta, siendo mayor la del país situado más al oriente. En bcasiones los meridianos geográficos no coinciden con los que sirven de límite o de separación de los husos porque tienen éstos que ajustarse a los límites de los páíses comprendidos dentro de cada huso. Hay también países que abarcan varios husos y que se ven por consecuencia obligados a utilizar horas diferentes dentro de un mismo país.

Situado México dentro del uso que tiene como meridiano central el de 90 grados al Oeste de Greenwich, tendrł como hora oficial la que corresponde a ese meridiomo. Según ésto comenzará el día oficial en todos los puntos de México cuando el Sol medio pase por el antimeridiama de los 90 grados. Lo hora media local diferirá de la hora oficial. Solamente habrá coincidencia entre una y otra en aquellos pueblas que estén situados en el meridiano de los noventa grados. La hora local 
de los pueblos situados a occidente será menor que la hora oficial y la diferencia entre una y otra será igual a la diferencia de longitudes. Si concemos pues la hora media local y la diferencia de longitudes con el meridiano de los sesenta grados nos será fácil pasar de la hora local a la hora oficial. Bastará sumar a la primera la diferencia de longitudes para cbtener la segunda.

Como los cuadrantes solares nos dan la hora solar verda. dera, para pasar a la hora oficial tendrémos que sumar, a la indicación del cuadrante, el valor de la ecuación de tiempo más el valor de la diferencia de longitudes entre el meridiano del emplazamiento del cuadrante y el meridiano de los sesenta grados. 